\title{
Aspectos obstétricos, psicossociais e sociodemográficos que podem potencializar risco para autismo nos primeiros nove meses de vida
}

\section{Obstetric, psychosocial and sociodemographic aspects that may increase risk for autism in the first nine months of life}

\author{
Antônia Motta Roth Jobim van Hoogstraten ${ }^{1}$, Ana Paula Ramos de Souza ${ }^{2}$, \\ Anaelena Bragança de Moraes ${ }^{3}$
}

http://dx.doi.org/10.11606/issn.2238-6149.v30i1p27-36

\begin{abstract}
Roth-Hoogstraten A, Souza APR, Moraes AB. Aspectos obstétricos, psicossociais e sociodemográficos que podem potencializar risco para autismo nos primeiros nove meses de vida. Rev Ter Ocup Univ São Paulo. 2019 jan.-abr.;30(1):27-36.

RESUMO: Objetivo: Analisar a associação entre presença de risco psíquico, a partir do questionário PREAUT, e fatores obstétricos, sociodemográficos e psicossociais, em bebês nascidos a termo e pré-termo entre um e nove meses de idade. Método: A amostra foi de 80 bebês, 25 nascidos pré-termo e 55 a termo avaliados a partir de entrevista semi-estruturada e aplicação do questionário PREAUT. Resultados: Houve associação entre presença de risco psíquico e o fato de a mãe cuidar sozinha do bebê, não apresentar uma atividade profissional, o bebê ser do sexo masculino, apresentar dificuldades alimentares como refluxo e engasgos, bem como não explorar o próprio corpo e o ambiente a sua volta. Conclusão: Houve associação entre presença de risco psíquico, a partir do questionário PREAUT e fatores obstétricos, sociodemográficos e psicossociais.
\end{abstract}

DESCRITORES: Determinantes sociais da saúde; Desenvolvimento infantil; Transtorno autístico.
Roth-Hoogstraten A, Souza APR, Moraes AB. Obstetric, psychosocial and sociodemographic aspects that may increase risk for autism in the first nine months of life. Rev Ter Ocup Univ São Paulo. 2019 Jan.-Apr.;30(1):27-36.

ABSTRACT: Objective: To analyze the association between presence of psychic risk, from the PREAUT grid and obstetric, sociodemographic and psychosocial factors, in term and preterm infants between one and nine months of age. Method: The sample consisted of 80 infants, 25 preterm and 55 term infants, who were evaluated using a semi-structured interview and the PREAUT grid. Results: There were association between presence of psychic risk and the fact that the mother cared for the baby all by herself, did not present a professional activity, the baby were male, presented eating difficulties such as reflux and gagging, as well as not exploring their own body or the environment around him. Conclusion: There were an association between the presence of psychic risk, based on the PREAUT grid and obstetric, sociodemographic and psychosocial factors.

KEYWORDS: Social determinants of health; Child development; Autistic disorder.

O presente artigo apresenta resultados parciais da dissertação "Sinais de risco psíquico em bebês na faixa etária de três a nove meses e sua relação com variáveis obstétricas, sociodemográficas e psicossociais". A pesquisa faz parte do Programa de Pós-Graduação em Distúrbios da Comunicação Humana da Universidade Federal de Santa Maria, UFSM, e recebeu financiamento da Coordenação de Aperfeicoamento de Pessoal de Nível Superior, CAPES.

1. Psicóloga, psicanalista, mestre e doutoranda pelo Programa de Pós-Gaduação em Distúrbios da Comunicação Humana, Universidade Federal de Santa Maria, UFSM. ORCID: https://orcid.org/0000-0002-1266-5072. Email: antoniamroth@gmail.com.

2. Fonoaudióloga, Pós-Doutora em Linguística (UFRGS), docente dos programas de Pós-Graduação em Distúrbios da Comunicação Humana e em Psicologia, Universidade Federal de Santa Maria, UFSM. ORCID: 0000-0003-4733-0632. Email: ana.souza@ufsm.br.

3. Doutora em Epidemiologia (UFSM), professora associada no Programa de Pós-Gaduação em Distúrbios da Comunicação Humana, Universidade Federal de Santa Maria, UFSM. ORCID: ORCID: 0000-0002-6578-0613. Email: anaelena@smail.ufsm.br.

Endereço para correspondência: Antonia Motta Roth Jobim van Hoogstraten. E-mail: antoniamroth@gmail.com. 


\section{INTRODUÇÃO}

$\mathrm{A}_{\text {possibilidade de identificar risco psíquico em }}^{\text {tualmente pesquisadores vem investigando a }}$ bebês a partir da qualidade das interações entre mãe (ou principal cuidador) e bebê, com especial foco na linguagem que compartilham, isto é, o manhês ou motherese. Esses estudos têm permitido observar sinais que identificam precocemente se o bebê está apresentando risco para autismo, pensando na necessidade de intervenção em tempo quando algo indica déficit na intersubjetividade primária ${ }^{1-4}$.

A responsividade do bebê ao manhês, ao olhar e ao toque, ancorada na relação afetiva com a mãe, incide sobre o desenvolvimento da capacidade de atenção compartilhada, de aprendizagem e de interação social. Entretanto, nos casos de bebês que receberam diagnóstico de autismo após três anos de idade, tal responsividade esteve consideravelmente diminuída ou ausente ${ }^{1,3}$. Isso se refletiu na menor presença de comportamentos intersubjetivos no segundo semestre de vida quando comparados a bebês em desenvolvimento típico ou mesmo com déficit intelectual ${ }^{3}$.

Em pesquisa recente ${ }^{1}$ foi avaliada a capacidade do questionário PREAUT (Prevention d'Autisme) instrumento oriundo da Pesquisa PREAUT (Programme Recherche Evaluation Autisme), desenvolvida na França por pesquisadores, com objetivo a avaliação de perturbações precoces da comunicação nos primeiros meses da vida de um bebê e que podem prever um distúrbio grave do desenvolvimento do tipo autístico. Na pesquisa o instrumento foi utilizado para prever autismo em 4.835 crianças acompanhadas até os 24 meses e avaliadas com o CHAT (Checklist for Autism Toddlers). Das 100 crianças que apresentaram resultado positivo para o CHAT, 45 receberam um diagnóstico no seguimento. E, dentre aqueles que receberam um diagnóstico, 22 não apresentaram qualquer alteração, 10 foram diagnosticados com autismo, sete com deficiência intelectual (ID), e seis tiveram outro transtorno do desenvolvimento. As pontuações do questionário PREAUT foram significativamente associadas ao status de risco de autismo médio e alto quando comparados aos resultados obtidos com a aplicação do CHAT aos 24 meses (odds ratio de 12,1 (IC 95\%: 3,0-36,8), $\mathrm{p}<0,001$, aos quatro meses e 38,1 (IC 95\%: $3,65-220,3), \mathrm{p}<0,001$, aos nove meses). Tais resultados indicam que o questionário PREAUT pode contribuir para a deteç̧ão muito precoce de risco para autismo e que sua combinação com o CHAT pode aumentar a acurácia de um diagnóstico precoce.

Tais resultados corroboram com a pesquisa ${ }^{5}$ que encontrou boa preditividade do questionário PREAUT, aplicado a bebês de nove meses diagnosticados com síndrome de West, quando comparado ao CHAT aos 24 meses. Os autores puderam confirmar a hipótese do questionário PREAUT de que, desde os primeiros meses de vida, os bebês posteriormente diagnosticados com autismo, apresentam dificuldades de responsividade ao outro e quase ausência de comportamentos intersubjetivos.

Considerando a especificidade do questionário PREAUT para detecção precoce de autismo ${ }^{5}$ é importante lembrar a dimensão neurobiológica implicada neste cenário. Haveria, nesses sujeitos, uma falha no desenvolvimento dos sistemas que programam o timing, a coordenação motora seriada, o controle prospectivo de movimentos e o controle da regulação afetiva das experiências ainda no período prénatal ${ }^{4}$. Por esse motivo, haveria ainda no primeiro ano de vida das crianças autistas a evidência de algumas consequências negativas, para além dos comportamentos intersubjetivos, relacionados a atividades básicas da vida diária, tais como sentar, engatinhar, arrastar-se e andar ${ }^{6,7}$.

Nesse contexto, salienta-se a influência de outros fatores biológicos para a incidência de presença de risco psíquico no bebê como, por exemplo, a prematuridade ${ }^{8}$. Para além da imaturidade orgânica, nos casos de prematuridade há que se considerar o problema dos efeitos ambientais. Isso acontece, por exemplo, quando há longos períodos de internação do bebê interferindo negativamente no estabelecimento da relação mãe-bebê essencial para a constituição psíquica do bebê. $\mathrm{E}$, nos casos em que o bebê apresenta alguma tendência congênita ao autismo essa tendência genética pode ser reforçada pelo ambiente iatrogênico da unidade de terapia intensiva e pela fragilidade física do bebê $\hat{e}^{9}$. Bebês prematuros e com risco psíquico tendem a ter mais déficits na integração sensorial, o que explica sintomas de hipersensibilidade e hipossensibilidade que levam esses bebês a assumirem posturas defensivas ${ }^{10}$.

Quanto aos fatores sociodemográficos, obstétricos e psicossociais e suas possíveis repercussões no desenvolvimento infantil, assinalam-se como fatores de risco socioambientais, toda a experiência adversa de vida ligada ao bebê e a sua família, tais como baixa escolaridade dos pais, estresse familiar, discórdia marital, doença psiquiátrica dos pais, baixo suporte social, baixa renda, práticas inadequadas de cuidado. Esses fatores interferem para agravar casos de risco para autismo e casos de risco para outras psicopatologias ${ }^{11-14}$.

Considerando que fatores ambientais e biológicos podem influenciar na geração de risco psíquico e ao desenvolvimento, este estudo objetiva analisar as possíveis associações entre risco para autismo, a partir do questionário PREAUT, e fatores sociodemográficos, obstétricos e psicossociais em um grupo de bebês acompanhados de um a nove meses de idade. O presente estudo apresenta resultados parciais da pesquisa de mestrado intitulada "Sinais de risco psíquico em bebês na faixa etária 
de três a nove meses e sua relação com variáveis obstétricas, sociodemográficas e psicossociais".

\section{PROCEDIMENTOS METODOLÓGICOS}

Esta pesquisa é do tipo quantitativa, de coorte prospectiva, pois acompanhou bebês prematuros e a termo ao longo dos nove primeiros meses de vida, enfocando a presença ou ausência de risco psíquico, a partir do questionário PREAUT ${ }^{15}$. Vincula-se a projeto de pesquisa realizado em uma Unidade Básica de Saúde e no Hospital Universitário de Santa Maria (HUSM), localizados no mesmo bairro de Santa Maria, Rio Grande do Sul, Brasil. Nesses dois ambulatórios, bebês nascidos pré-termo e a termo foram acompanhados ao longo dos 24 primeiros meses de vida.

\section{Participantes}

A amostra total contou com 80 bebês, sendo 55 bebês nascidos a termo ( 29 meninos e 26 meninas) e 25 bebês nascidos pré-termo com idade gestacional inferior a 37 semanas (sendo 11 meninos e 14 meninas). Os bebês prematuros foram avaliados considerado a idade corrigida. Entre os nascidos pré-termo tem-se 21 bebês com prematuridade tardia e idade gestacional entre 32 e 36 semanas e seis dias (224 a 258 dias); e, 4 bebês com prematuridade moderada e idade gestacional entre 28 e 31 semanas e 6 dias (196 e 223 dias).

Foram excluídos todos os bebês que apresentaram qualquer espécie de síndrome genética (síndrome de Down, $\mathrm{X}$-frágil, etc.), lesões neurológicas como encefalopatia crônica não progressiva, ou déficits sensoriais como surdez ou déficit visual. Esse critério de exclusão foi garantido nos bebês prematuros a partir das avaliações que o seguimento de prematuros do hospital universitário realizada ainda antes da alta hospitalar. Os bebês nascidos a termo foram avaliados por pediatras da unidade básica de saúde e encaminhados para exames neurológicos em caso de suspeita de alteração biológica. Neste caso, foram excluídos da amostra.

\section{Aspectos éticos}

Esta pesquisa, conforme normas regulamentadas pela Resolução 466/2012 (BRASIL Resolução n 466, de 12 de dezembro de 2012), passou por Aprovação do Comitê de Ética e Pesquisa em Saúde (CEP) da UFSM e por autorização da Secretaria Municipal de Saúde, inserida no projeto maior intitulado "Análise Comparativa do Desenvolvimento de Bebês Prematuros e a Termo e sua Relação com Risco Psíquico: da detecção à intervenção", autorizado em maio de 2014 no CEP-UFSM sob número CAAE: 28586914.0.0000.5346.
A pesquisa foi realizada entre maio de 2015 e maio de 2016 e não ofereceu riscos físicos ou morais para os participantes, uma vez que os exames realizados pela equipe são procedimentos não invasivos, caracterizados por entrevistas e filmagens que podem apresentar desconforto mínimo pelo tempo de execução.

No primeiro contato com as díades foram expostas e atendidas as demandas éticas da pesquisa. Foi entregue a todas as mães que aceitaram participar da pesquisa um Termo de Consentimento Livre e Esclarecido constando os objetivos da pesquisa, os riscos envolvidos, a participação voluntária, o sigilo quanto a sua identidade e o seu direito de desistir do processo em qualquer momento do estudo.

\section{Instrumentos de coleta}

\section{Entrevista semi-estruturada}

O roteiro da entrevista semi-estruturada foi construído a partir do roteiro já utilizado ${ }^{12}$, que sofreu acréscimos para ampliação da investigação de fatores psicossociais, sociodemográficos e obstétricos. O roteiro foi aplicado, no primeiro encontro, por meio de perguntas apresentadas à mãe e/ou quem faça a função materna. Nos encontros seguintes, tais questões eram abordadas para se verificar as mudanças ocorridas na rotina da família. Quanto aos fatores sociodemográficos, psicossociais e obstétricos, foram considerados:

- Fatores sociodemográficos: idade materna e paterna; estado civil materno e paterno; escolaridade materna e paterna; atividade profissional e situação profissional materna e paterna; número de filhos da mãe e do pai; participação do pais nos cuidados do bebê, idade dos irmãos; presença de outro cuidador e seu parentesco com o bebê; número de pessoas que residem na casa; renda mensal familiar; tipo de residência;

- Fatores psicossociais: quem permanece mais tempo com a criança; a mãe (ou aquele que exerce a função materna) possui algum suporte social; o bebê possui contato com outras crianças; demonstra preferir algum brinquedo; quando acordado, explora diferentes posições; de que forma chama a mãe; ela chama o bebê; como ele responde; a mãe conversa com ele; em que momentos; algo o incomoda; como ele informa; como é a sua rotina; ele participa da rotina familiar; onde dorme e com quem; a mãe enfrentou alguma crise situacional no período da gestação, nos primeiros dias após o nascimento do bebê e/ou após passados alguns meses; histórico de doença mental na família; como o pai está se sentindo em relação ao bebê. 
- Fatores obstétricos: nascimento pré-termo ou a termo; realizou pré-natal; número de consultas pré-natal; iniciou o pré-natal a partir de quando; intercorrências na gestação; ruptura prematura da membrana (bolsa); uso de medicamento(s) na gestação; uso de drogas; uso de álcool; uso de fumo; infecções intrauterinas; histórico de aborto; histórico de parto prematuro; planejamento da gestação do bebê avaliado; desejo materno de engravidar; tipo de parto realizado; peso do bebê ao nascer; Apgar 1'e Apgar 5'; etnia do bebê; intercorrências neonatais; período de internação do bebê em UTI neonatal; uso de ventilação mecânica; uso de medicação ototóxica; hiperbilirrubinemia; procedimento terapêutico utilizado; tipo de aleitamento; leite artificial utilizado; dificuldade alimentar; transição alimentar; uso de chupeta.

\section{Questionário PREAUT}

O questionário PREAUT apresenta como objetivo avaliar o fechamento do circuito pulsional, a partir de sinais tanto no nível corporal quanto na voz materna e interação com o bebê. Pra tal, são avaliados dois sinais fenomênicos a partir da relação intersubjetiva da díade mãe-bebê, são eles²: o bebê procura "se fazer" olhar por sua mãe (ou substituto) na ausência de qualquer solicitação dela; e, o bebê procura suscitar trocas prazerosas com sua mãe (ou com seu substituto) na ausência de qualquer solicitação dela, chamando sua atenção ou fazendo gracinhas, por exemplo.

$\mathrm{Na}$ presente pesquisa, inicialmente, os sinais foram observados a partir da interação mãe-bebê e depois da interação entre a pesquisadora e as díades. Salienta-se que, nos casos em que a mãe não interagia com o seu bebê no período de avaliação, a pesquisadora solicitou que a mesma conversasse com o seu bebê, tal como faz em casa. Foi atribuído um valor para cada resposta às perguntas apresentadas no questionário PREAUT. Quando o somatório era 15, considerava-se ausente o risco para o autismo e se inferior a 15 presença de risco fosse ele grave (inferior a 5) ou moderado (entre 5 e 15). A seguir, no quadro 1, apresentase o questionário PREAUT.

Quadro 1 - Questionário PREAUT utilizados na pesquisa e verificados na faixa etária do $4^{\circ}$ e do $9^{\circ}$ mês

\section{O bebê procura olhar para você?}

a) Espontaneamente

b) Quando você fala com ele (protoconversação)

2. O bebê procura "se fazer" olhar por sua mãe (ou pelo substituto dela)?

a) $\mathrm{Na}$ ausência de qualquer solicitação da mãe, vocalizando, gesticulando ao mesmo tempo em que a olha intensamente.

b) Quando ela fala com ele (protoconversação)

\begin{tabular}{|l|l|}
\hline Sim & 4 \\
Não & 0 \\
\hline Sim & 1 \\
Não & 0
\end{tabular}

Realizar a segunda parte do questionário se o somatório das questões 1 e 2 for inferior à 5

3. Sem qualquer estimulação de sua mãe (ou de seu substituto)

a) Ele olha para sua mãe (ou para seu substituto)

b) Ele sorri para sua mãe (ou para seu substituto)

c) O bebê procura suscitar uma troca prazerosa com sua mãe (ou seu substituto), por exemplo, se oferecendo ou estendendo em sua direção os dedos do seu pé ou da sua mão?

\begin{tabular}{|l|l|}
\hline Sim & 8 \\
Não & 0 \\
\hline Sim & 2 \\
Não & 0 \\
\hline
\end{tabular}

4. Depois de ser estimulado por sua mãe (ou pelo seu substituto)

a) Ele olha para sua mãe (ou substituto)

b) Sorri para sua mãe (ou para seu substituto)

c) O bebê procura suscitar a troca jubilatória com sua mãe (ou com seu substituto), por exemplo, se oferecendo ou estendendo em sua direção os dedos do seu pé ou da sua mão?

\begin{tabular}{|l|l|}
\hline Sim & 1 \\
Não & 0 \\
\hline Sim & 2 \\
Não & 0 \\
\hline Sim & 4 \\
Não & 0 \\
\hline \multicolumn{2}{|l|}{} \\
\hline Sim & 1 \\
Não & 0 \\
\hline Sim & 2 \\
Não & 0 \\
\hline Sim & 4 \\
Não & 0 \\
\hline
\end{tabular}

Fonte: Laznik et al. (1998) 


\section{Procedimentos}

Após aceitarem participar da pesquisa e assinarem o TCLE, as mães responderam a entrevista inicial semiestruturada. Alguns dados foram confirmados no cartão de saúde do bebê. Depois do primeiro contato, foram previstos outros dois encontros. Por fim, o quarto encontro foi previsto para oferecer a devolutiva aos responsáveis do bebê, bem como encaminhamentos para intervenção quando necessário, a partir de atendimento individual especializado ou grupos de musicalização.

Nos encontros para avaliação foram realizadas filmagens da interação mãe-bebê ao longo de nove minutos. A interação entre mãe e bebê foi filmada em setting montado em uma sala silenciosa, em que a díade ficava sobre um tapete de EVA, em frente a um espelho. Aos quatro meses o bebê ficava em um bebê conforto de costas para o espelho e a mãe de frente para o bebê. Aos nove meses, o bebê já podia sentar sem apoio tendo mais liberdade para movimentar-se.

Aos quatro meses a mãe era convidada a cantar por três minutos, conversar por outros três minutos e brincar com animal de borracha com o bebê por outros três minutos. A interação foi registrada por meio de duas câmeras digitais, uma colocada há um metro de distância em visão lateral da díade e outra há dois metros que permitia uma visão frontal do bebê e o reflexo materno no espelho. Aos nove meses, embora a mãe fosse orientada a cantar, conversar e brincar com o bicho, a interação poderia ser menos esquemática tendo em vista o fato de o bebê se deslocar pela sala.

O estabelecimento de faixa etária de coleta, a partir do questionário PREAUT e os procedimentos realizados estão descritos a seguir:

- Faixa etária de 1 mês - no teste do Pezinho primeiro contato com a díade mãe-bebê, convite para participar da pesquisa, assinatura do TCLE e entrevista inicial;

- Faixa etária de 4 meses - entre 3 meses e 1 dia a 4 meses e 29 dias - aplicação do questionário PREAUT, entrevista continuada e filmagem da díade;

- Faixa etária de 9 meses - entre 8 meses e 1 dia e 9 meses e 29 dias - aplicação do questionário PREAUT, entrevista continuada e filmagem da díade.

\section{Análise dos dados}

Para a análise das variáveis avaliadas, após a coleta dos dados as informações foram reunidas em uma planilha do Excel e atualizadas de acordo com cada uma das fases avaliadas. Para a análise estatística, utilizaram-se os aplicativos computacionais Statistica 9.1 e PASW///. Em relação aos testes aplicados, efetuou-se a análise de regressão logística dos resultados obtidos com o questionário PREAUT, variável dependente relacionada à presença de risco psíquico, cujos resultados foram confrontados com as demais variáveis independentes cuja associação foi calculada a partir do modelo logístico chamado odds ratio (OR ajustado), considerando a característica de interesse: apresenta ou não sinais de risco psíquico.

A regressão logística considerou um nível de significância de $10 \%$ com o objetivo de verificar o desfecho "presença de risco" que se associava a fatores sociodemográficos, obstétricos e psicossociais coletados nas entrevistas. Para tal, foi considerada como "presença de risco": bebês que apresentaram uma pontuação do questionário PREAUT inferior a 15 pontos no que concerne a avaliação das faixas etárias de 4 e 9 meses.

\section{RESULTADOS}

A partir do questionário PREAUT, os resultados da regressão logística com o desfecho "presença de risco", em bebês prematuros e a termo, nas duas faixas etárias avaliadas, podem ser visualizados nas tabelas 1 e $2 . \mathrm{Na}$ Tabela 1, observa-se a frequência de bebês a termo e prematuros com questionário PREAUT alterados nas duas avaliações. Os resultados demonstram um grande número de bebês na primeira fase com os sinais alterados $(52,5 \%$ da amostra). Percebe-se que a ocorrência de presença de risco psíquico em bebês prematuros foi $11 \%$ mais alta do que aqueles bebês nascidos a termo.

Segundo o questionário PREAUT, aos nove meses, o percentual de bebês com risco psíquico caiu consideravelmente $(21,25 \%)$. Em relação às pontuações iguais ou inferiores a 5 , na primeira fase surgem oito casos $(10 \%)$ e nenhum na segunda fase. Desses oito casos referidos da primeira fase, três dos seis bebês nascidos a termo e um dos dois prematuros foram avaliados com idade inferior a quatro meses, o que pode sugerir que ainda estivessem muito imaturos no momento inicial da avaliação.

Na Tabela 2, são apresentados, respectivamente, as frequências, os percentuais, as razões de risco (OR) e seus respectivos intervalos de confiança para cada uma das fases avaliadas, considerando as variáveis sociodemográficas, psicossociais e obstétricas que foram significativas no modelo de regressão logística múltipla considerando o resultado obtido no questionário PREAUT. 
Roth-Hoogstraten A, et al. Aspectos obstétricos, psicossociais e sociodemográficos. Rev Ter Ocup Univ São Paulo. 2019 jan./abr.;30(1):27-36.

Tabela 1 - Frequência dos bebês a termo e prematuros com questionário PREAUT alterados nas duas avaliações

\begin{tabular}{|c|c|c|c|c|c|c|c|c|c|c|c|c|}
\hline & \multirow{2}{*}{$\begin{array}{c}\text { Número de } \\
\text { bebês com risco }\end{array}$} & \multicolumn{11}{|c|}{ Pontuação total dos bebês com risco } \\
\hline & & 13 & 12 & 11 & 10 & 9 & 8 & 7 & 6 & 5 & 3 & 2 \\
\hline \multicolumn{13}{|c|}{ Questionário PREAUT } \\
\hline Prematuros $(n=25)$ & $15(60 \%)$ & & & & 1 & & 2 & 9 & 1 & 1 & & 1 \\
\hline A termo $(n=55)$ & $27(49 \%)$ & & 2 & & & 1 & 2 & 15 & 1 & 2 & 2 & 2 \\
\hline Total $(n=80)$ & $42(52,5 \%)$ & & 2 & & 1 & 1 & 4 & 24 & 2 & 3 & 2 & 3 \\
\hline \multicolumn{13}{|c|}{$\begin{array}{l}\text { Questionário PREAUT } \\
\text { Fase III ( }\left(9^{\circ} \text { mês }\right)\end{array}$} \\
\hline Prematuros $(n=25)$ & $6(24 \%)$ & & & 1 & & & & 4 & 1 & & & \\
\hline A termo $(n=55)$ & $11(20 \%)$ & 1 & & & & 1 & & 9 & & & & \\
\hline Total $(n=80)$ & $17(21,25 \%)$ & 1 & & 1 & & 1 & & 13 & 1 & & & \\
\hline
\end{tabular}

Sem risco = Somatório total do questionário PREAUT igual à 15; Com risco = Somatório total do questionário PREAUT inferior à 15. Fonte: Autores.

Tabela 2 - Variáveis sociodemográficas, psicossociais e obstétricas em relação ao PREAUT avaliado nas duas fases

\begin{tabular}{|c|c|c|c|c|c|}
\hline & \multicolumn{2}{|c|}{ PREAUT } & \multirow{2}{*}{ OR Aj } & \multirow{2}{*}{ IC $90 \%$} & \multirow{2}{*}{ p-valor } \\
\hline & Sem risco $(\%)$ & Com risco (\%) & & & \\
\hline \multicolumn{6}{|c|}{3 meses -4 meses e 29 dias } \\
\hline \multicolumn{6}{|c|}{ Atividade profissional } \\
\hline Trabalha/estudante & $26(61,90 \%)$ & $16(38,10 \%)$ & 1 & & \\
\hline Dona de casa & $12(31,58 \%)$ & $26(68,42 \%)$ & 3,384 & $1,517-7,550$ & 0,012 \\
\hline \multicolumn{6}{|c|}{ Dificuldade alimentar } \\
\hline Não & $29(50,88 \%)$ & $28(49,12 \%)$ & 1 & & \\
\hline Sim & $9(39,13 \%)$ & $14(60,87 \%)$ & 3,453 & $1,361-8,762$ & 0,029 \\
\hline \multicolumn{6}{|c|}{8 meses a 9 meses e 29 dias } \\
\hline \multicolumn{6}{|c|}{$\begin{array}{l}\text { Mãe com cônjuge que lhe auxilia nos } \\
\text { cuidados do bebê }\end{array}$} \\
\hline Sim & $55(80,89 \%)$ & $13(19,11 \%)$ & 1 & & \\
\hline Não & $8(66,67 \%)$ & $4(33,33 \%)$ & 3,490 & $1,046-11,649$ & 0,088 \\
\hline \multicolumn{6}{|c|}{$\begin{array}{l}\text { Bebê busca experimentar diferentes } \\
\text { posições }\end{array}$} \\
\hline Sim & $54(81,82 \%)$ & $12(18,18 \%)$ & 1 & & \\
\hline Não & $9(56,25 \%)$ & $5(31,25 \%)$ & 3,328 & $1,046-10,593$ & 0,088 \\
\hline \multicolumn{6}{|l|}{ Prematuridade } \\
\hline Não & $44(80,00 \%)$ & $11(20,00 \%)$ & 1 & & \\
\hline Sim & $19(76,00 \%)$ & $6(24,00 \%)$ & 4,001 & $1,347-11,883$ & 0,036 \\
\hline \multicolumn{6}{|l|}{ Sexo do bebê } \\
\hline Feminino & $35(88,00 \%)$ & $5(12,00 \%)$ & 1 & & \\
\hline Masculino & $28(70,00 \%)$ & $12(30,00 \%)$ & 3,241 & $1,161-9,050$ & 0,06 \\
\hline
\end{tabular}

Sem risco $=$ Somatório total do questionário PREAUT igual à $15 ;$ Com risco = Somatório total do questionário PREAUT inferior à $15 ;$ OR Aj= odds ratio ajustado; IC=Intervalo de Confiança. Fonte: Autores.

Os resultados do modelo de regressão apresentados na tabela 2 ilustram como fatores que estatisticamente influenciaram o desfecho "presença de risco" para o questionário PREAUT (decorrente de um somatório inferior a 15 pontos), na faixa etária dos quatro meses, as variáveis: presença de atividade profissional materna e presença de dificuldade alimentar do bebê.

Foi considerado fator de risco a mãe não ter nenhuma atividade profissional, seja trabalhar e/ou estudar. O fator descrito sugere que os bebês de mães donas de casa possuem 
duas vezes mais chance de apresentar questionário PREAUT com somatório inferior a 15 quando comparados aos bebês de mães que exercem alguma atividade profissional. É importante ressaltar que no período da avaliação dos quatro meses, essa variável sociodemográfica considerou como sendo mães socialmente ativas aquelas que estavam em licença maternidade, bem como as que tinham abandonado o emprego após o nascimento do filho, mas pretendiam retomá-lo após o sexto mês do bebê.

Quanto às variáveis obstétricas, verificou-se em relação a dificuldades alimentares, que os bebês a termo $(n=8)$ e com PREAUT alterados na faixa etária do $4^{\circ}$ mês, apresentaram queixas de refluxo $(n=5)$ e de engasgo $(n=3)$. Em relação aos bebês prematuros $(n=6)$ que obtiveram PREAUT alterados na mesma faixa etária, as queixas também foram de refluxo $(n=4)$ e de engasgo $(n=2)$. Tais resultados sugerem que bebês com dificuldades alimentares apresentaram duas vezes mais risco de apresentar ao menos um dos dois sinais PREAUT avaliados ausentes na primeira fase quando comparados aos bebês que não apresentaram dificuldades para alimentar-se.

Quanto à avaliação do questionário PREAUT na faixa dos nove meses, obtiveram-se como principais fatores associados ao desfecho "presença de risco": a prematuridade, o sexo do bebê, a mãe possuir um cônjuge que lhe auxilia nos cuidados do bebê e a capacidade do bebê de explorar o ambiente a sua volta.

Verificou-se como fator de proteção, o fato da mãe possuir cônjuge que auxilia nos cuidados do bebê. Nesse sentido, evidenciou-se que mães que possuem cônjuge, seja através de casamento civil ou união estável, possuem duas vezes menos chance de seus bebês apresentarem ao menos um sinal PREAUT ausente, na faixa etária dos nove meses, em relação àquelas mães que não possuíam cônjuge que olhe oferece suporte.

No rol de aspectos psicossociais, evidenciou-se como fator de proteção, o bebê explorar diferentes posições corporais, bem como o ambiente e os objetos a sua volta. Nesse sentido, os bebês que se movimentavam livremente explorando seu corpo e o ambiente ao seu redor, apresentavam duas vezes menos chance de apresentaram o questionário PREAUT alterados na faixa dos nove meses, em relação àqueles que não experimentavam diferentes posições.

Por fim, salienta-se que dentre os aspectos obstétricos, a prematuridade e o sexo do bebê foram fatores que incidiram diretamente sobre os resultados na avaliação do questionário PREAUT na faixa etária dos nove meses. Bebês que nasceram prematuramente tiveram três vezes mais chance de apresentar ao menos um sinal PREAUT ausente em relação aos bebês a termo. Além disso, bebês do sexo masculino apresentaram duas vezes mais chance de apresentar ao menos um dos dois sinais avaliados no PREAUT ausentes, quando comparados aos bebês do sexo feminino.

\section{DISCUSSÃO}

Os resultados da presente pesquisa sugerem que, $o$ questionário PREAUT é sensível para detectar risco psíquico desde os quatro meses ${ }^{1-5}$. Todavia, pode-se verificar que diversos fatores concorrem para a existência de tal desfecho, inclusive alguns relacionados ao próprio bebê, como seu sexo, dificuldades alimentares, prematuridade, sua iniciativa em experimentar diferentes posições e explorar o ambiente a sua volta, bem como fatores maternos, como a presença de atividade profissional e de apoio do cônjuge.

Em relação aos aspectos biológicos, percebe-se, tal como encontrado na literatura, que a prematuridade ${ }^{8,9}$ representou um fator de risco à constituição psíquica, tendo em vista que bebês prematuros apresentaram três vezes mais chances de apresentar somatório inferior a 15 no questionário PREAUT aos 9 meses do que aqueles nascidos a termo. Além disso, percebe-se que na amostra inicial, constituída por 25 bebês prematuros e 55 a termo, na fase I houve um percentual bastante superior de bebês prematuros $(60 \%)$ com questionário PREAUT alterados em comparação aos bebês a termo (49\%). Esse percentual reduz na segunda avaliação quanto à incidência de bebês com risco passando a $24 \%$ nos casos dos bebês prematuros e $20 \%$ nos casos dos bebês a termo. Percebe-se que, apesar da redução, a ocorrência de presença de risco permaneceu superior nos casos dos bebês nascidos com idade gestacional inferior a 37 semanas.

Tais resultados confirmam a afirmação de que o nascimento prematuro pode representar fator de risco psíquico ao bebê e de crise psicológica na família ${ }^{9}$, bem como no desenvolvimento de habilidades sensoriomotoras ${ }^{4,6,7,10}$. Ressalta-se que tais fatores podem contribuir para a evolução autista do bebê $\hat{e}^{1,5}$.

Embora a relação entre prematuridade e risco psíquico não tenha sido muito evidenciada na literatura, a associação com alterações motoras é frequentemente citada, pois é conhecida a relação entre idade gestacional inferior a 32 semanas e peso menor ou igual a 1500 gramas em relação à resultados inferiores no desenvolvimento neuropsicomotor ${ }^{8,16}$.

O que estes estudos afirmam, a partir do olhar da psicologia, foi reforçado por estudo recente com 554 bebês prematuros ${ }^{17} \mathrm{em}$ que $55(10 \%)$ casos apresentaram sinais de risco de autismo. $\mathrm{O}$ alto percentual de bebês prematuros que apresentaram sinais de risco psíquico também pode estar associado a questões de oxigenação durante período neonatal ${ }^{13}$. 
Outro fator obstétrico estatisticamente significativo para o desfecho "presença de risco" foi o sexo do bebê. Os resultados sugerem que bebês do sexo masculino apresentaram duas vezes mais chance de apresentar somatório final do questionário PREAUT inferior a 15. Esse resultado confirma estudos ${ }^{14}$ anteriores que demonstraram que os meninos apresentam maior frequência de transtornos do desenvolvimento do que as meninas. Essas evidências confirmam os resultados preliminares da pesquisa PREAUT na França em que dos 24 bebês que apresentaram risco de evolução para autismo em um dos exames, houve uma prevalência do sexo masculino $(n=16)$ em relação ao feminino $(\mathrm{n}=8)^{15}$.

Outras variáveis independentes que apresentaram correlação estatisticamente significativa na regressão logística foram presença de cônjuge participativo na vida da díade e atividade laborial materna. Os resultados demonstraram que bebês cujas mães não possuem o suporte do cônjuge, bem como não tem atividade profissional (seja ela estudar e/ou trabalhar) apresentaram menor pontuação no questionário PREAUT.

Esses resultados indicam que a mãe ou aquele que assume a função materna possuir uma relação estável, consensual, que potencializa seu desejo de cuidar de seu bebê são fatores de proteção contra o risco à constituição psíquica de seu bebê. Mães cujo cônjuge the auxilia nos cuidados do bebê possuem duas vezes mais chance de apresentar bebês com os dois sinais PREAUT presentes e o estabelecimento do terceiro tempo pulsional, na faixa etária dos nove meses, quando comparados aos bebês de mães solteiras e sozinhas no cuidado. Esse resultado reforça a importância da presença de um cônjuge desde o nascimento do bebê, promovendo sustentação à mãe para que ela possa exercer sua função e atuando como terceiro na relação mãe-bebê produzindo a separação necessária à constituição psíquica do bebê. Esse resultado confirma resultados encontrados ${ }^{12}$.

Outro resultado protetivo ao desenvolvimento do bebê foi o fato de a mãe possuir alguma atividade profissional. Em relação aos sinais PREAUT avaliados no quarto mês, tem-se que a mãe não apresentar alguma atividade profissional, seja estudar e/ou trabalhar, aumentou em duas vezes a chance de seu filho apresentar sinais de risco psíquico. Esse resultado também foi encontrado em outro estudo ${ }^{12} \mathrm{em}$ que a perspectiva profissional materna parece promover seu bem-estar e melhores condições para cuidar de seu bebê.

Quanto às dificuldades alimentares evidenciadas nos bebês, pôde-se verificar que os bebês a termo $(n=8)$ e com questionário PREAUT alterados na faixa etária do $4^{\circ}$ mês, apresentaram queixas de refluxo $(n=5)$ e de engasgo $(n=3)$.
Em relação aos bebês prematuros $(n=6)$ que obtiveram questionário PREAUT alterados na mesma faixa etária, quatro apresentaram queixa de refluxo e dois de engasgo. Tais resultados sugerem que bebês com dificuldades alimentares apresentaram duas vezes mais risco psíquico na primeira fase dos questionários PREAUT do que aqueles bebês que não apresentaram dificuldades para alimentar-se.

Percebe-se que a presença de dificuldade alimentar como o refluxo, mostrou-se como fator importante a ser considerado nos casos de autismo ${ }^{18}$. Os autores sugerem que crianças autistas frequentemente apresentam problemas digestivos. Este fato parece explicar a associação encontrada com o questionário PREAUT e presença de refluxo e engasgo nesta pesquisa. Também uma pesquisa com o instrumento IRDI encontrou associação entre dificuldades na transição alimentar e presença de risco psíquico ${ }^{19}$.

Apresentou-se como fator de proteção, o bebê experimentar diferentes posições corporais e explorar livremente o ambiente e os objetos. Nesse sentido, bebês que se movimentavam livremente explorando seu corpo e o ambiente ao seu redor sob a supervisão materna ou de outro responsável, apresentavam duas vezes menos chance apresentarem o questionário PREAUT alterados na faixa dos nove meses, em relação àqueles que não experimentavam diferentes posições. Esses resultados confirmam evidências de alterações psicomotoras em bebês que desenvolveram autismo ${ }^{6,7}$ e também estudos que associaram sintomas psicomotores ao risco psíquico na avaliação por meio do instrumento Indicadores de Risco/Referência do Desenvolvimento Infanti ${ }^{20}$.

\section{CONCLUSÃo}

A partir do objetivo proposto no presente artigo de verificar possíveis associações entre risco psíquico e fatores obstétricos, psicossociais e sociodemográficos em bebês de um a nove meses, a partir do questionário PREAUT pode-se concluir que diversos fatores concorrem para a existência de risco psíquico. Dentre as variáveis independentes que apresentaram associação estatisticamente significativa para o desfecho "presença de risco", tem-se como fatores obstétricos e biológicos do bebê: bebês do sexo masculino, nascimento prematuro, a presença de dificuldades alimentares, tais como engasgos e refluxo gastro-esofágico. Dentre os fatores sociodemográficos, as variáveis que apresentaram associação estatisticamente significativa com o desfecho presença de risco foram: a mãe não possuir apoio de um cônjuge e não possuir uma atividade profissional. Por fim, dentre os fatores psicossociais que apresentaram associação estatisticamente significativa com o desfecho presença de risco, tem-se: o 
Roth-Hoogstraten A, et al. Aspectos obstétricos, psicossociais e sociodemográficos. Rev Ter Ocup Univ São Paulo. 2019 jan./abr.;30(1):27-36.

bebê ser capaz de explorar o ambiente a sua volta a partir de diferentes posições corporais.

O trabalho evidencia a necessidade de adoção de medidas protetivas para as populações de risco que abrange uma política de acompanhamento pré-natal e pós-natal mais efetiva para evitar nascimentos prematuros, melhorar as condições do parto e oferecer condições de inserção laboral para a mãe e auxílio à família no cuidado com o bebê. Isso sugere que se deve aumentar a eficiência das políticas previstas pelo Sistema Único de Saúde brasileiro, mas que infelizmente não estão implementadas a contento na realidade da cidade na qual a pesquisa foi realizada.

Participação dos autores: Roth-Hoogstraten A - Responsável pela coleta e análise dos dados da pesquisa e redação do artigo. Souza APR - Responsável pela orientação da coleta e análise dos dados, bem como da redação do artigo. Moraes $A B$ - Responsável pela orientação da análise estatística dos dados e revisão da escrita do artigo.

\section{REFERÊNCIAS}

1. Olliac B, Crespin G, Laznik MC, Cherif Idrissi El Ganouni O, Sarradet JL, Bauby C, Dandres AM, Ruiz E, Bursztejn C, Xavier J, Falissard B, Bodeau N, Cohen D, Saint-Georges C. Infant and dyadic assessment in early community-based screening for autism spectrum disorder with the PREAUT grid. PLoS ONE. 2017;12(12):e0188831. doi: 10.1371/ journal.pone.0188831.

2. Laznik MC. Diversos olhares sobre o autismo. In: Jerusalinsky A, editor. Dossiê autismo. São Paulo: Instituto Langage; 2015. p.56-61.

3. Saint-Georges C, Chetouani M, Cassel R, Apicella F, Mahdhaoui A, Muratori F, Laznik MC, Cohen D. Motherese in interaction: at the cross-road of emotion and cognition? (a systematic review). PLoS ONE. 2013;8(10):e78103. doi: 10.1371/journal.pone.0078103.

4. Trevarthen C, Delafield-Butt J. Autism as a development disorder in intentional movement and affective engagement. Front Integr Neurosc. 2013;17(7):49. doi: 10.3389/ fnint.2013.00049.

5. Ouss L, Saint-Georges C, Robel L, Bodeau N, Laznik MC, Crespin GC, Chetouani M, Bursztejn C, Golse B, Nabbout $\mathrm{R}$, Desguerre I, Cohen D. Infant's engagement and emotion as predictors of autism or intellectual disability in West syndrome. Eur Child Adolesc Psychiatry. 2014;23:143-9. doi:10.1007/s00787-013-0430-x.

6. Muratori F. O diagnóstico precoce no TEA: guia prático para pediatras. Salvador: Núcleo Interdisciplinar de Intervenção Precoce da Bahia; 2014.

7. Esposito G, Venuti P, Maestro S, Muratori F. An exploration of symmetry in early TEA spectrum disorders analysis of lying. Brain Dev. 2009;31:131-8. doi: 10.1016/j.braindev.2008.04.005.

8. Serenius F, Källén K, Blennow M, Ewald U, Fellman V, Holmström G, Lindberg E, Lundqvist P, Maršál K, Norman M,
Olhager E, Stigson L, Stjernqvist K, Vollmer B, Strömberg B; EXPRESS Group. Neurodevelopmental outcome in extremely preterm infants at 2.5 years after active perinatal care in Sweden. JAMA. 2013;309(17):1810-20. doi: 10.1001/jama.2013.3786.

9. Vanir C. Os mecanismos operantes no desenvolvimento psíquico do bebê prematuro e os riscos eventuais de patologia. In: Brusnel MC, Melgaço R, editores. O bebê e as palavras: uma visão transdisciplinar sobre o bebê. São Paulo: Instituto Langage; 2013. p.49-58.

10. Beltrami VH, Moraes AB, Souza APR. Perfil sensorial e sua relação com risco psíquico, prematuridade e desenvolvimento motor e de linguagem por bebês de 12 meses. Rev Ter Ocup Univ São Paulo. 2018;29(1):8-18. doi: 10.11606/ issn.2238-6149.v29i1p8-18.

11. Roth-Hoogstraten AM, Moraes AB, Souza APR. A complementaridade entre sinais PREAUT e IRDI na análise de risco psíquico aos nove meses e sua relação com idade gestacional. CoDAS. 2018;30(5):e20170096. doi: 10.1590/2317-1782/20182017096.

12. Crestani A, Mattana F, Moraes A, Souza APR. Fatores socioeconômicos, obstétricos, demográficos e psicossociais como risco ao desenvolvimento infantil. Rev CEFAC. 2013;15(4):847-56. doi: 10.1590/S1516-18462013000400013.

13. Sandin S, Lichtenstein P, Kuja-Halkola R, Larsson H, Hultman CM, Reichenberg A. The familial risk of autism. JAMA. 2014;311(17):1770-7. doi: 10.1001/jama.2014.4144.

14. Schjolberg S, Eadie P, Zachrisson HD, Oyen AS, Prior M. Predicting language development at age 18 months: data from the Norwegian Mother and Child Cohort Study. J Dev Behav Pediatr 2011;32(5):375-83. doi: 10.1097/ DBP.0b013e31821bd1dd.

15. Cullere-Crespin G, Parlato-Oliveira E. Projeto PREAUT. In: Jerusalinsky A, editor. Dossiê autism. São Paulo: Instituto Langage; 2015. p.436-55. 
Roth-Hoogstraten A, et al. Aspectos obstétricos, psicossociais e sociodemográficos. Rev Ter Ocup Univ São Paulo. 2019 jan./abr.;30(1):27-36.

16. Fernandes LV, Goulart AL, Santos AM, Barros MC, Guerra CC, Kopelman BI. Neurodevelopmental assessment of very low birth weight preterm infants at corrected age of 18-24 months by Bayley III scales. J Pediatr. 2012;88(6):471-8. doi: 0021-7557/12/88-06/471.

17. Stephens B, Vohr, B. Neurodevelopmental outcome of the premature infant. Pediatr Clin North Am. 2009;56(3):631-46. doi: 10.1016/j.pcl.2009.03.005.

18. Laznik MC, Burnod Y. O ponto de vista dinâmico neuronal sobre as intervenções precoces. In Kupfer MC, Szejer M. (Eds.) Luzes sobre a clínica e o desenvolvimento de bebês: novas pesquisas, saberes e intervenções. São Paulo: Instituto Langage; 2016. p.18-35.

19. Vendruscolo J, Bolzan GM, Crestani AH, Souza APR, Moraes AB. A relação entre o aleitamento, a transição alimentar e os indicadores de risco para o desenvolvimento infantil. Disturb Comun. 2012;24(1):41-52. doi: 10.1590/S151618462013000400013.

20. Kupfer MC, Bernardino L. As relações entre construção da imagem corporal, função paterna e hiperatividade: reflexões a partir da pesquisa IRDI. Rev Latinoam Psicopatol Fundam. 2009;12(1):45-58. doi: 10.1590/S1415-47142009000100004. 